\title{
Modes of interaction between nanostructured metal and a conducting mirror as a function of separation and incident polarization
}

\author{
F. Bonnie, M.D. Arnold, G.B. Smith* and A.R. Gentle \\ Physics and Advanced Materials, Institute of Nanoscale Technology \\ University of Technology, Sydney, PO Box 123, Broadway NSW 2007 Australia
}

\begin{abstract}
The optical resonances that occur in nanostructured metal layers are modulated in thin film stacks if the nanostructured layer is separated from a reflecting conducting layer by various thicknesses of thin dielectric. We have measured and modeled the optical response of interacting silver layers, with alumina spacer thickness ranging from a few $\mathrm{nm}$ to $50 \mathrm{~nm}$, for s- and p-polarized incident light, and a range of incident angles. Standard thin film models, including standard effective medium models for the nanostructured layer, will break down for spacer thickness below a critical threshold. For example, with polarisation in the film plane and some nano-islands, it may occur at around $10 \mathrm{~nm}$ depending on spacer refractive index. Of particular interest here are novel effects observed with the onset of percolation in the nanolayer. Hot spot effects can be modified by nearby mirrors. Other modes to consider include (a) a two-particle mode involving a particle and its mirror image (b) A Fano resonance from hybridisation of localized and de-localised plasmon modes (c) a Babinet's core-(partial) shell particle with metal core-dielectric shell in metal (d) spacing dependent phase modulation (e) the impact of field gradients induced by the mirror at the nano-layer.
\end{abstract}

Keywords: metal island films, percolation, nanolayer-spacer-mirror, hybrid modes, surface plasmons

\section{INTRODUCTION}

Metal Island Films (MIF) and their evolution into conducting or percolating networks as deposited metal mass increases have a long history [1] and were one of the first examples studied in detail where localised surface plasmon resonances (LSPs) could be optically excited. Understanding optical responses as the metal islands began to link up, was a bigger challenge with key new insights emerging quite recently [2,3]. An admix of two broad classes of LSP and an emergent Drude type response were all present in the transition zone [3], until only a Drude response occurred at sufficient mass and it evolved until all voids were filled. A major attraction has been the variety of spectral responses that resulted both at solar wavelengths and in the thermal infra-red, which could be further tuned and amplified by filling the nanovoids with dielectric. This scope for optical engineering of absorptance, which covers not only spectral, but also polarisation and angle of incidence responses, can be dramatically changed yet again when the MIF or percolating network is part of a three layer system as shown in figure 1a, b. A mirror layer is added with a variety of dielectric thicknesses separating the nano and mirror layers. Such systems have become one key element in an emerging major new field, thermal engineering with meta-materials [4]. Of particular interest is simultaneous control of both solar radiation and thermally emitted radiation from any surface. This involves optical engineering with thin films over a very broad band from 0.3 $\mu \mathrm{m}$ to $35 \mu \mathrm{m}$. One example has been known for some time, solar selective absorbers, which have very high solar absorptance and low thermal emittance, as needed for efficient solar thermal collectors. Many variations arise however, depending on the particular application. Solar control with insulating low emittance windows, white or various colored coatings with high solar reflectance and either very high black body emittance or emittance only over wavelengths where the atmosphere is transparent, involve many spectral variants [5-7]. A core aim is night sky cooling without high daytime gains [8]. Another example of thermal emission over restricted bands within the Planck radiation spectrum, at a given temperature includes, high emission at temperatures around $1000^{\circ} \mathrm{C}$, at wavelengths where a solar cell works best $[4,9]$ (called thermo-photovoltaics-TPV). It is however, essential in such systems to also have very low emission at other Planck wavelengths, so that most energy is channeled to the cell's optimum band. This is another more stringent form of spectral selectivity.

Nanostructured Thin Films VI, edited by Tom G. Mackay, Akhlesh Lakhtakia, Yi-Jun Jen, Motofumi Suzuki, Proc. of SPIE Vol. 8818, 881809 - (c) 2013 SPIE · CCC code: 0277-786X/13/\$18 · doi: 10.1117/12.2022794 
(a)

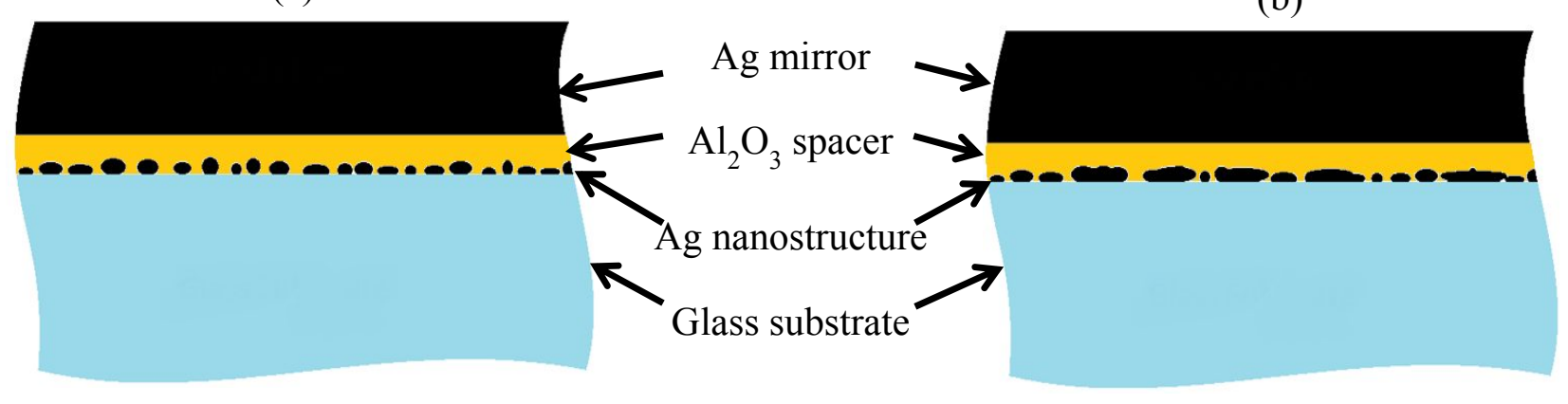

Figure 1. Schematic of two final 3-layer structures of interest. Silver is in black and the intermediate oxide layer is in yellow. (a) Initial isolated islands some close (b) Partial island linking and percolation. Hot spots are found in (a) and (b). Mass thickness of silver is larger in (b) than in (a).

There is much to consider in the theoretical modeling of these systems, even with no scattering. The few detailed studies to date, have tended to focus on one or two of these processes only. A baseline question is, can one model the experimental data with standard thin film (STF) models? If each layer in a stack acts as a planar layer with the complex indices as found when in isolation, then one can, but if any are directly coupled by evanescent fields so that hybrid new modes arise, an integrated (i.e. non-STF) approach is needed. Types of direct coupling that may occur include (i) a local two particle mode between a nano-island multi-pole and its mirror image, essentially a two particle LSP mode (ii) a delocalized mode with LSPs coupling to surface plasmon polaritons (SPP) on the mirror, which is a class of Fano resonance. Such coupling can be strongly polarization dependent but this work and related theory indicates very close approach is needed, 10 to $12 \mathrm{~nm}$ for low index $(\mathrm{n}=1.4-1.5)$ spacers [10], and lower for higher index $(\mathrm{n}=1.9$ to 2.4) insulators. A study, which in common with this one, considered cross-coupling in the percolation zone did find an anomalously large absorptance, but attributed it to a Fano type coupling at a spacer thickness of $40 \mathrm{~nm}$ [11]. This is unlikely from reference [10], and this study clearly shows that at $40 \mathrm{~nm}$ amplification of absorption near percolation is due simply to phase modulation, and that STF's apply. Generic mathematical forms for resonance can often seem to fit data, but unless fitting parameters are linked to images of physical nanostructure that have evanescent fields extending far enough for hybrid effects, fitting parameters will not have a physical basis. Very strong resonances do however, occur in Ag films as percolating networks develop [3, 12].

If the dielectric spacer is a high index material it will inhibit inter-layer coupling by reducing the reach of the evanescent field compared to its extent in air. Hybrid modes are further complicated for partially percolating films where "anomalous" LSPs involving hot spots exist [3] and arise in up to three distinct ways. These may be more likely to hybridize because the initiating local resonance can be much stronger than those on isolated islands, enabling stronger evanescent coupling. Anomalous local modes may arise from (i) isolated islands coupled to a local network [3] (ii) hotspots within a network, due to sharp local variations in spacing between network strands [12] (iii) the "mirror" layer following the contours of the underlying nanostructure, which leads to a Babinet's core-shell or metal core-in-metal shell nanoparticle resonances $[13,14]$. Each case has an identifiable class of nanostructures, so that careful studies of the nanostructure are needed to check if a given resonant process is possible.

A final issue arising with mirrors present and thick enough spacers that evanescent coupling is absent, is worth consideration. The ability to use a 3-layer thin film model may not always work without modification, if partial cavity (or Fabry-Perot) modes are formed by reflectance between the mirror and the nano-layer. That is the nano-layer in the presence of the mirror, may behave differently to the same layer in isolation. A driving force for such a change could be the field profile at the edge of a cavity mode for some spacings and nanostructures. For example, while a uniform effective medium may have been fine initially, one (n, k) pair may no longer describe the composite. Some explicit modeling and experiment is underway to investigate this issue.

Given this diversity of issues we focus in this report on the two major variable changes (i) nanostructure in the thin $\mathrm{Ag}$ layer (ii) spacer thickness. The focus is on inter-comparisons of experimental optical data to provide insight into the 
structural points, at which new physical mechanisms and spectral effects become apparent, where resonant absorption maximizes and thin film models applying for no mirror, have problems with a mirror present. Base data used thus has spacer present, with and without a mirror. From the no-mirror data, the complex indices of the base nanocomposite and the thickness of the $\mathrm{Al}_{2} \mathrm{O}_{3}$ insulator above the composite (which becomes the spacer thickness once a mirror is added), can be found without concern for hybrid or cavity effects. Then it is possible to see if these indices persist with the mirror present. The evolution of optical data with geometric changes supported by imaging yields significant insights into, which of many possible phenomena are present. We will only touch here on one hybrid aspect, namely when 3layer thin film models work and start to fail, or are inconsistent with the [two layer/ no mirror] models. The effect of the mirror on absorption strength through the percolation zone is also of interest, given the presence of anomalous modes within the Ag nanolayer.

\section{EXPERIMENTAL DETAILS}

Each layer is magnetron sputter coated. A silver target and DC power supply is used for the two metal containing layers, and an alumina target and RF supply for the spacer layer. A rotating substrate stage, set to receive flux from the two targets, was used to increase thickness uniformity, and it was calibrated for silver after 60 seconds using transmittance spectral data at different regions of the slide. Mass deposition rates in each layer were pre-calibrated. These were a starting guide to model thickness, but final thicknesses of the nano-layer and spacer layer were set using optical modeling of each layer alone and checked against the combination of nano-layer and spacer layer. Substrate material and cleanliness strongly influences the initial growth of thin silver films and the masses needed for percolation. For the base layer three depositions were carried out with mass thicknesses of silver of 5,6 and $7 \mathrm{~nm}$, which were found to span the percolation threshold, from single island and small chains dominating to porous network dominance. Super white glass slides were used to follow structural evolution with manageable thickness differences. Six alumina thicknesses were then added with nominal thicknesses ranging from $10 \mathrm{~nm}$ to $50 \mathrm{~nm}$. The $\mathrm{Al}_{2} \mathrm{O}_{3}$ spacer thickness as expected, was slightly thinner than the stand alone layer expected from calibration, as initially, some is used to fill residual voids in the nanolayer. This difference decreases as Ag mass thickness increases as this leads to a decrease in initial void content. The thinly deposited $\mathrm{Al}_{2} \mathrm{O}_{3}$ has two important roles (a) it fills the pores in the base silver layer which amplifies and shifts SPRs (b) it allows control of the dielectric nano-gap and hence coupling between the two metal layers once a mirror is added.

First the comparison of spectral responses for normal incidence, when the dielectric spacer is present without the mirror and after the mirror is added to the same two layer structure provides valuable insights. Then we consider how the three layer responses changes, as the spacing of the mirror from the nanostructured metal, varies from $10 \mathrm{~nm}$ up to $50 \mathrm{~nm}$. As normal incidence fields are in the film plane to probe effects of polarization normal to this plane spectral results and thin film modeling with the original optical constants for p-polarised oblique incidence have also been studied for a range of spacer thicknesses. Induced plasmonic based fields then have a component normal to the metal plane..

The main new insights from these studies turned out to be (i) the mirror's impact on the optical responses in the transition percolation zone in comparison to that of island only layers; (ii) the use of 3-layer systems of the form [nano$\mathrm{metal} / \mathrm{spacer} / \mathrm{metal}$-mirror] to tune absorptance over a wide range. Spectral control in applications which involve energy production using the sun or for energy efficiency are needed [5] (iii) that near flat spacers with index near 2.0 need to be less than $10 \mathrm{~nm}$ to observe inter-layer coupling and the breakdown of standard thin film models. Limited aspects only have been studied [15]. Item (iii) has been recently been theoretically and experimentally examined for gold [10], but the spacer was a polyelectrolyte or a set of amine-terminated alkanethiols, with spacer thickness in effect fixed by the number of carbon atoms in the chain. Such layers have much lower indices than our inorganics, enabling wider spread of the evanescent field. Plasmonic coupling is still only easily observable below $10 \mathrm{~nm}$ of spacer. Thus coupling at $10 \mathrm{~nm}$ here is unlikely but may occur due to other effects, including non-smooth metal mirror layer exciting Babinet's coreshell local resonances, or from strong local hot-spots in the percolation zone. 


\section{OPTICAL MODELS}

Optical modeling to find complex indices $\mathrm{n}, \mathrm{k}$ for the nano-composite layer formed from growing noble metal films then adding an oxide over-coat, has often been successfully carried out, and the same approach is used here. Fitting with a set of Lorentz oscillators of the form of equation (1) works since the generic plasmonic absorption processes, even if each involves multiple individual resonances, do not significantly overlap. The three generic absorption processes of interest before the mirror is added are (i) isolated islands of Ag (ii) "hot spots" in the developing Ag network (iii) de-localised conduction in a nano-porous Ag network. If any of these three break down or change significantly when the mirror is added due to hybrid plasmonic effects, the original thin film model based on this layer will break down in well-defined frequency ranges. That is, in some wavelength ranges, we then expect the stack to behave as a single entity. A simple modeling approach to having a wavelength dependent mix of STF behavior and hybrid effects is under consideration. Here we focus only on examining if the single composite layer $\mathrm{n}, \mathrm{k}$ values continue to apply at all solar wavelengths and spacer thicknesses down to $10 \mathrm{~nm}$ once the mirror is added.

$$
\varepsilon(\omega, t)=\varepsilon_{\infty}(t)+\sum_{i=1}^{3} \frac{A_{i}(t)}{\omega_{i}^{2}-\omega^{2}-i \gamma_{i}(t) \omega}
$$

Equation (1) can implicitly include inter-band absorption in Ag if desired, but as they are centred in the UV, it is acceptable to add their impact into the constant $\varepsilon_{\infty}$ in this study. $A_{i}(t)$ in units $(\mathrm{eV})^{2}$ is the spectral weight of the plasmonic response centred at $\omega_{i}$ and depends on mass thickness $t$, which controls the relative weights of processes (i), (ii), (iii). For (iii) $\omega_{i}=0$ and Drude like response occurs. Its spectral weight is however not that of bulk Ag, which is $\omega_{P}^{2}\left(\omega_{P}=\right.$ plasma frequency), but increases towards $\omega_{P}{ }^{2}$ as $\mathrm{Ag}$ mass increases and nano-voids fill. $\gamma_{i}(t)$ is the usual conduction electron relaxation rate (in $\mathrm{eV}$ ) for the Drude term at each mass.

The standard Fresnel thin film multilayer models are used to fit optical data in the context of the WVASE ellipsometric and spectrophotometric software, including fine adjustment of thicknesses when needed for best fit. The oscillator parameters for the nano-layers acquired before adding the mirror layer appear in table 1 in the results section.

\section{RESULTS}

To demonstrate changes as Ag mass thickness varies, three sets of normal incidence spectral reflectance plots are compared, one for each different Ag mass. Within each mass thickness set, relevant experimental and modeled data are presented. Modeling parameters used are listed in table 1 below. The first example set is in fig. 2 for the thinnest $5 \mathrm{~nm}$ mass $\mathrm{Ag}$ layer, covered with six $\mathrm{Al}_{2} \mathrm{O}_{3}$ thicknesses, with and without a mirror. The experimental absorption peak in 2(a) is dominated by isolated $\mathrm{Ag}$ island nanoparticle resonance absorption, peaking near $670 \mathrm{~nm}$. The second plot in $2 \mathrm{~b}$ is of a 3-layer thin film model, using the complex optical constants $\mathrm{n}$, $\mathrm{k}$, for the composite layer, derived from joint fitting of front and back reflectance and transmittance on the 2-layer system before the mirror was added. Two of these inputs with no mirror are plotted in 2(c). Comparing this experimental reflectance and transmittance of the no-mirror film in (c), to the other two plots (a) and (b), highlights the main changes that occur on adding the mirror. With no mirror, peak resonant absorptance lies in the narrow range 0.40 and 0.51 , while when the mirror is added it is much enhanced and spread out as a function of spacer thickness, and covers the range between 0.48 and 0.84 . It can be seen that the thin film models in the (b) plots, provide an excellent description of the experimental (a) data in the $5 \mathrm{~nm}$ case, even down to $\mathrm{Al}_{2} \mathrm{O}_{3}$ spacings of $10 \mathrm{~nm}$. Hybrid coupling of any type including a Fano resonance is clearly not present, otherwise normal thin film models would fail. In part one could attribute lack of coupling to having the field within the plane at normal incidence, which might limit the coupling strength. Oblique incidence p-polarised light was used to explore the polarisation direction issue with dipoles pointing towards the mirror, rather than parallel to it. For all three sets we found that standard thin film models still worked quite well with the original optical constants for $60^{\circ}$ oblique incidence and ppolarisation. Thus coupling is absent or very weak when the alumina spacer is above $10 \mathrm{~nm}$ thick for any polarisation. The reflectance in (c) falls slightly, but not as much as expected for an island only sample. However, this $5 \mathrm{~nm}$ sample is unusual in that the nano-layer appears to have a high contribution from hot-spots, but no net Drude type contribution. 
Table 1. Parameters used in equation (1) to jointly fit spectral transmittance, back and front spectral reflectance, for the nano-silver layer in the percolation zone when over-coated with alumina. From these the complex indices n, $\mathrm{k}$ for the nanocomposite $\mathrm{Ag} / \mathrm{Al}_{2} \mathrm{O}_{3}$ were derived.

\begin{tabular}{|lccc|}
\hline Lorentz Oscillator Parameters & \multicolumn{3}{c|}{ Ag Mass Thickness (nm) } \\
\hline & $\mathbf{5}$ & $\mathbf{6}$ & $\mathbf{7}$ \\
\hline$\varepsilon(\infty)$ & $2.26 \pm 0.04$ & $2.59 \pm 0.03$ & $2.90 \pm 0.02$ \\
\hline $\mathrm{A}($ hot-spot $)\left(\mathrm{eV}^{2}\right)$ & $19.5 \pm 0.4$ & $12.3 \pm 1.6$ & $11.0 \pm 0.7$ \\
\hline$\gamma_{\mathrm{i}}$ (hot-spot) $(\mathrm{eV})$ & $1.53 \pm 0.03$ & $1.17 \pm 0.08$ & $1.03 \pm 0.04$ \\
\hline$\omega_{\mathrm{i}}$ (hot-spot $(\mathrm{eV})$ & $0.96 \pm 0.01$ & $0.96 \pm 0.02$ & $0.92 \pm 0.01$ \\
\hline $\mathrm{A}($ Ag Island) $(\mathrm{eV})$ & $18.8 \pm 0.3$ & $16.2 \pm 1.1$ & $12.8 \pm 0.5$ \\
\hline$\gamma_{\mathrm{i}}($ Ag island) $(\mathrm{eV})$ & $1.01 \pm 0.01$ & $1.11 \pm 0.03$ & $0.95 \pm 0.02$ \\
\hline$\omega_{\mathrm{i}}$ (Ag island) $(\mathrm{eV})$ & $1.86 \pm 0.01$ & $1.70 \pm 0.01$ & $1.54 \pm 0.01$ \\
\hline $\mathrm{A}($ Drude $)\left(\mathrm{eV}{ }^{2}\right)$ & N/A & $14.6 \pm 0.3$ & $17.2 \pm 0.2$ \\
\hline$\gamma($ Drude $)(\mathrm{eV})$ & N/A & $0.47 \pm 0.02$ & $0.39 \pm 0.01$ \\
\hline
\end{tabular}

Close approaching particles and small gaps, which are found in images, may be enough to produce the hot spots. Indeed this sample's image shows many locally linked up particles and is interesting in that it still doesn't have a noticeable Drude response. An explanation is given by Earp and Smith [3], where it is shown that a conducting Drude response begins when network chains are of order half a wavelength long, which is well before dc conductivity indicates percolation due to its typical electrode spacings. The images of this film indicate linking has not yet reached that threshold. Thus, only hot spot and isolated particle resonances are found. The hot spot resonance peak is centered at $1,300 \mathrm{~nm}(0.96 \mathrm{eV})$, but is also widest in this layer (at $1.5 \mathrm{eV})$. Its isolated particle resonance is at $660 \mathrm{~nm}(1.9 \mathrm{eV})$, and also broad. The breadth of these peaks is associated with the greater complexity and variance in the local nanostructure at the threshold of percolation. This complexity, and with it peak widths, decrease as more Ag mass is added. A similar situation arises in figure 3 for the $6 \mathrm{~nm} \mathrm{Ag} \mathrm{mass,} \mathrm{with} \mathrm{some} \mathrm{important} \mathrm{differences.} \mathrm{Lower} \mathrm{hot} \mathrm{spot} \mathrm{spectral} \mathrm{weight,} \mathrm{some}$ drop in isolated particle contribution and a red shift from the $5 \mathrm{~nm}$ position, to $720 \mathrm{~nm}(1.7 \mathrm{eV})$ for its peak, but of most significance clear evidence of the onset of a Drude response. The latter's spectral weight of $15(\mathrm{eV})^{2}$, is however, weak and well below that of bulk silver of $\omega_{\mathrm{P}}{ }^{2}=80(\mathrm{eV})^{2}$. This is due to a combination of nano-voids within the conducting Ag network, and the spectral weights of resonating residual islands and resonating hot spots. ESEM (Environmental Scanning Electron Microscope) images comparing $5 \mathrm{~nm}$ and $6 \mathrm{~nm}$ films, show that networks have extended sufficiently to support optically induced free carrier response in the $6 \mathrm{~nm}$ case. 

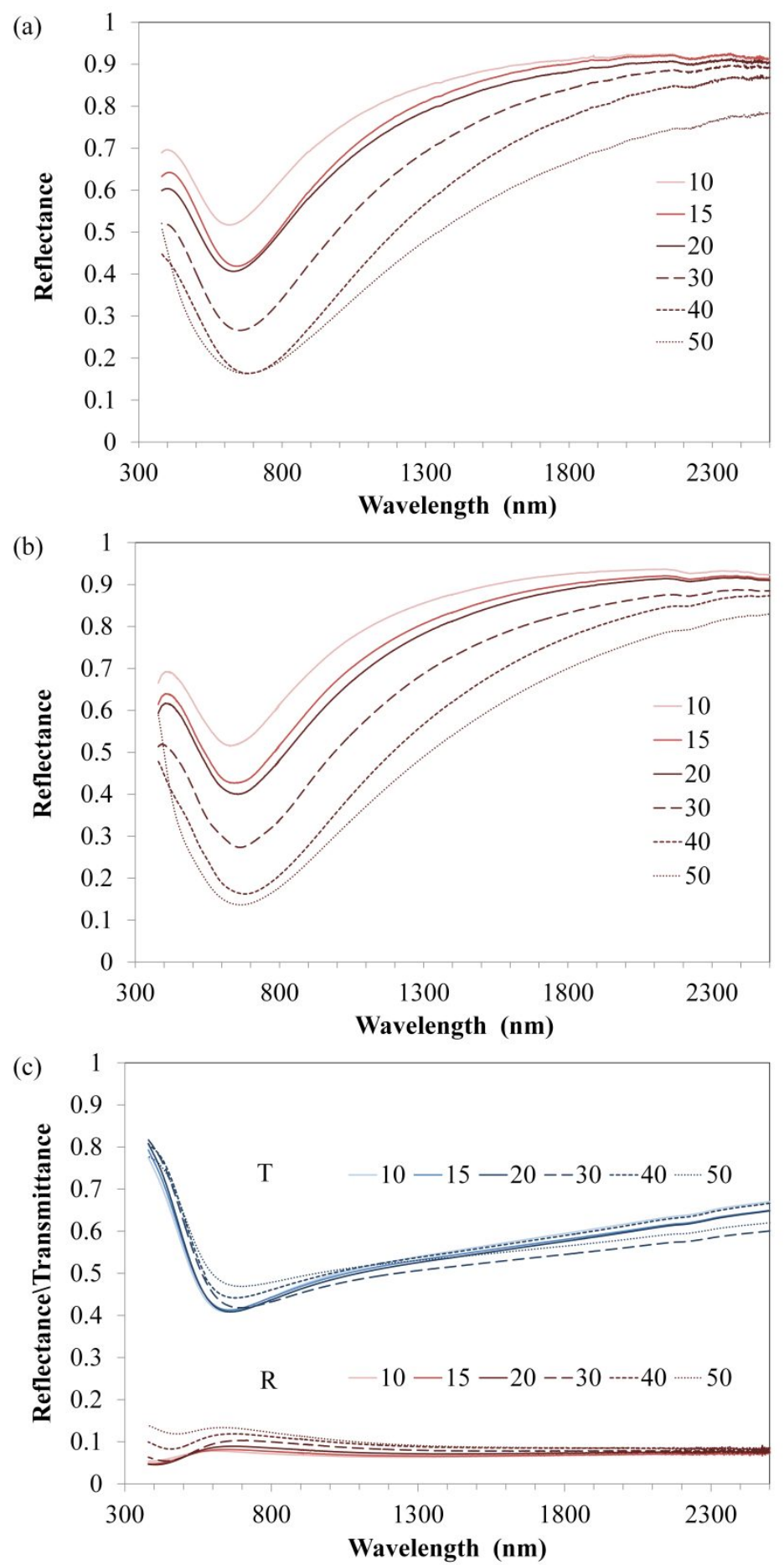

Figure 2. Spectral reflectance of $5 \mathrm{~nm}$ Ag layer coated with the same six $\mathrm{Al}_{2} \mathrm{O}_{3}$ thicknesses (a) Data with a mirror layer as measured (b) Modeled with a mirror layer using indices for the $5 \mathrm{~nm} \mathrm{Ag} / \mathrm{Al}_{2} \mathrm{O}_{3}$ layer (c) Data before a mirror is added and includes transmittance. 

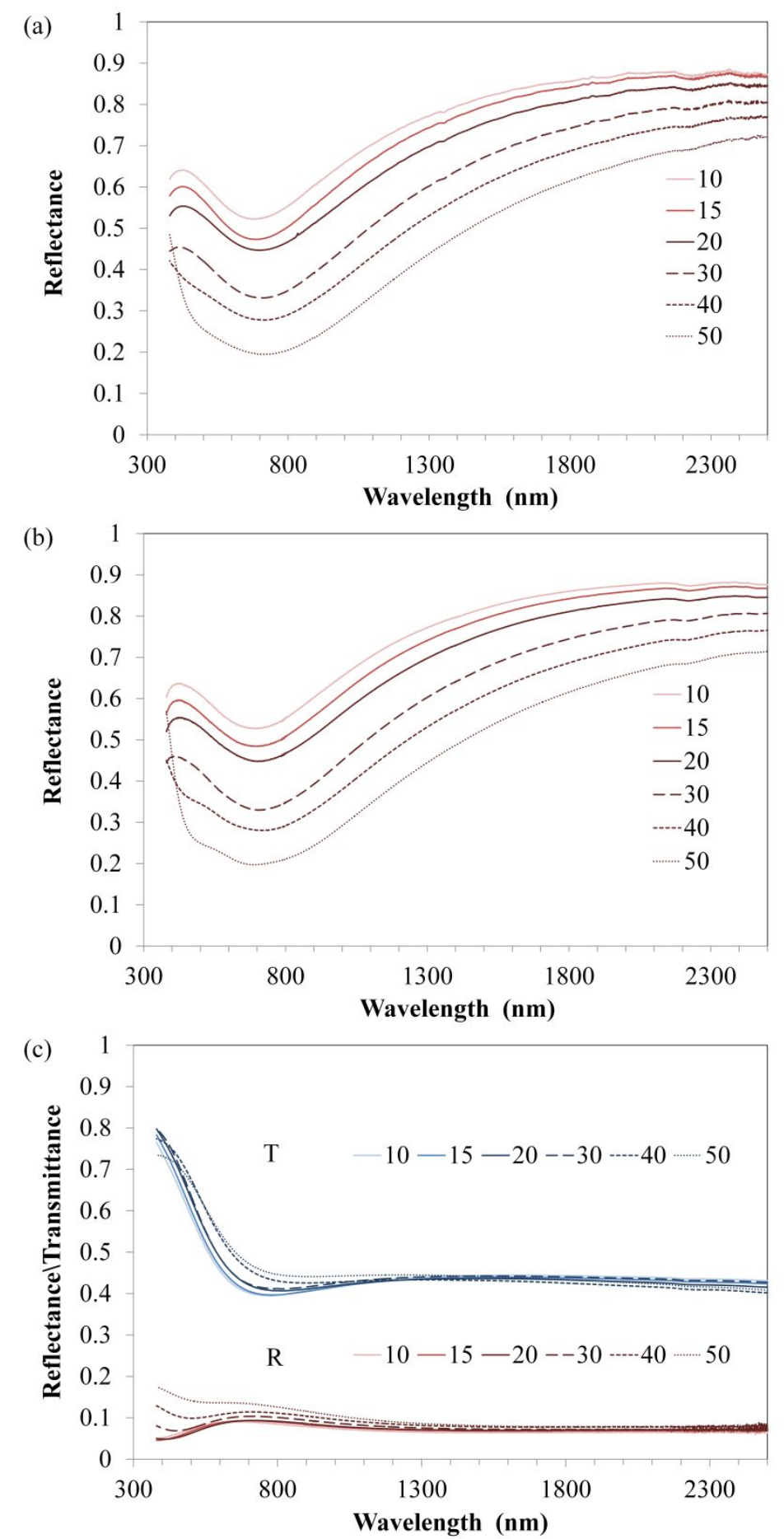

Figure 3. Spectral reflectance of $6 \mathrm{~nm}$ Ag layer coated with the same six $\mathrm{Al}_{2} \mathrm{O}_{3}$ thicknesses (a) With a mirror layer as measured (b) Modeled with a mirror layer using indices for the $6 \mathrm{~nm} \mathrm{Ag} / \mathrm{Al}_{2} \mathrm{O}_{3}$ layer (c) Data before a mirror is added and includes transmittance.

Figure 4 shows the data, models and no-mirror responses for the $7 \mathrm{~nm}$ case. For the thinnest spacers, modeled and experimental plots, in this case do not quite match up as well as in the $5 \mathrm{~nm}$ and $6 \mathrm{~nm}$ cases. A number of interesting qualitative differences compared to $5 \mathrm{~nm}$ and $6 \mathrm{~nm}$ responses also occur at all spacer thicknesses. With no mirror, isolated island resonances are further red shifted to $1.54 \mathrm{eV}(800 \mathrm{~nm})$ and the hot spot contribution is further reduced 
though still significant. The two localised plasmonic absorption modes still dominates, but the Drude response has strengthened to an $\mathrm{A}_{\text {Drude }}$ value of $17(\mathrm{eV})^{2}$. Once a mirror is added, simple thin film models continue to work well for spacer thicknesses from $50 \mathrm{~nm}$ to $20 \mathrm{~nm}$, while spectral response with a mirror is qualitatively similar to its thinner $\mathrm{Ag}$ counterparts. The 10 and $15 \mathrm{~nm}$ spacers provide much less absorption and higher reflectance than 10 and $15 \mathrm{~nm}$ spacers
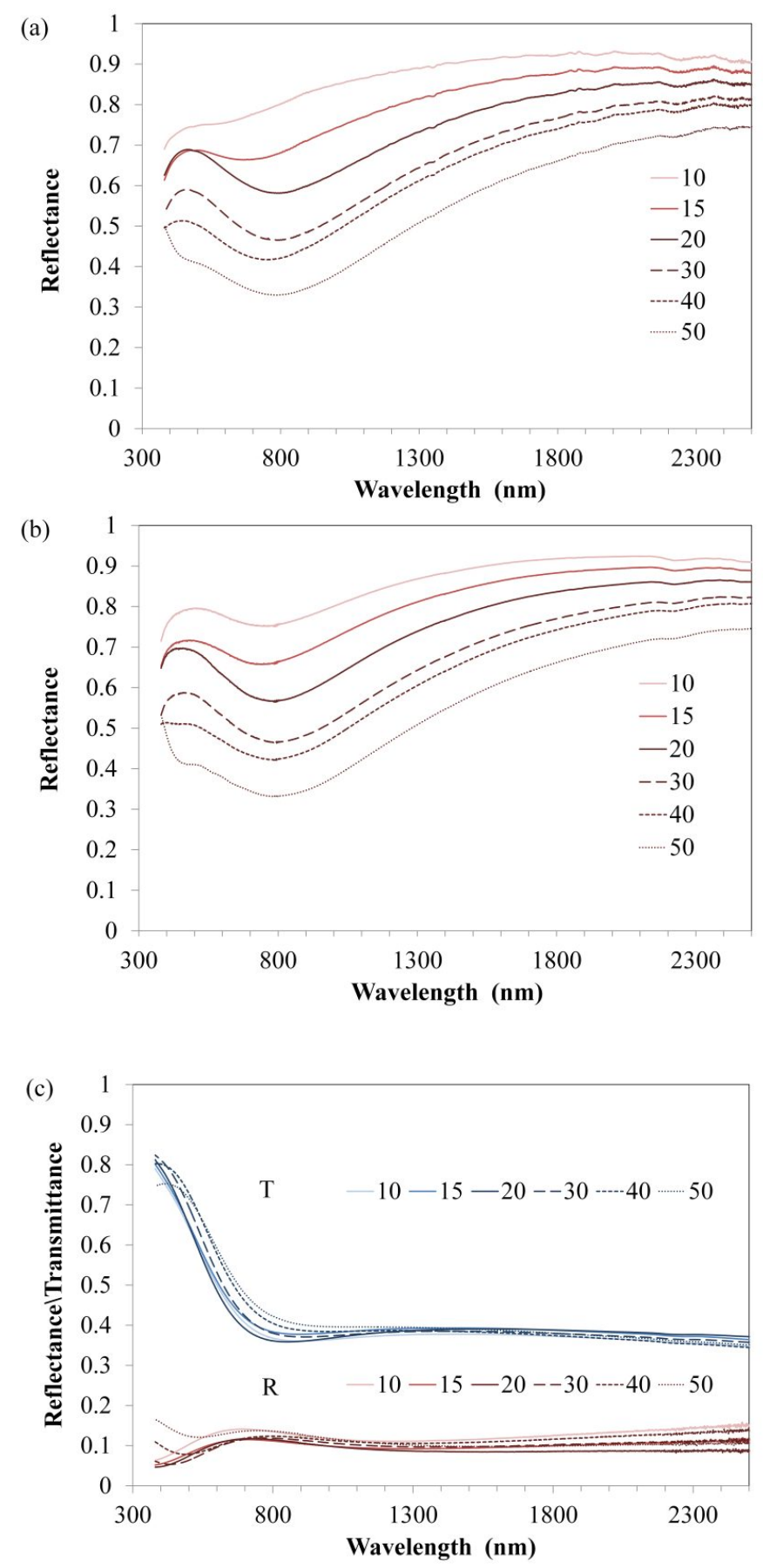

Figure 4. Spectral reflectance of $7 \mathrm{~nm} \mathrm{Ag}$ layer coated with the same six $\mathrm{Al}_{2} \mathrm{O}_{3}$ thicknesses (a) Data with a mirror layer as measured (b) Modeled with a mirror layer using indices for the $7 \mathrm{~nm} \mathrm{Ag} / \mathrm{Al}_{2} \mathrm{O}_{3}$ layer (c) Data before a mirror is added and includes transmittance. 
on $5 \mathrm{~nm}$ and $6 \mathrm{~nm}$ films, in both experiment and models. Models do not precisely fit observed final spectral shape and show lower reflectance, hence higher absorptance in the range $700 \mathrm{~nm}$ to $1200 \mathrm{~nm}$. The no-mirror data has absorption in this range near $50 \%$ as do the alumina coated $5 \mathrm{~nm}$ and $6 \mathrm{~nm} \mathrm{Ag}$ films. What is interesting is that with the mirror, the lower masses still absorb from $50 \%$ to $35 \%$ for 10 and $15 \mathrm{~nm}$ spacers, while at $7 \mathrm{~nm}$ absorptance with the same spacing has dropped to $30 \%$ to $20 \%$. Thin film models approximately agree with the largest discrepancy at around $1000 \mathrm{~nm}$ where they overestimate absorptance by $\sim 8 \%$. This wavelength range is where the hot spots have most influence. The thin spacer cases are the only ones where a hint emerges that simple thin film models may need modifying when a mirror is present.

The anomalous "hot spot" resonance peak required for best fit, unlike the island peak is very fixed in location for all three Ag masses at $0.96 \pm 0.2 \mathrm{eV}$. This has also been observed in our earlier work [2,3]. In contrast, the island peak red shifts from $1.9 \mathrm{eV}$ (5 nm Ag mass) to $1.7 \mathrm{eV}$ (6 nm Ag mass) and finally to $1.5 \mathrm{eV}$ (7 $\mathrm{nm} \mathrm{Ag}$ mass).

\section{DISCUSSION}

The sets of experimental data together indicate that with $\mathrm{Al}_{2} \mathrm{O}_{3}$ as a spacer, spectral response is very sensitive to its thickness in the range $10 \mathrm{~nm}$ to $50 \mathrm{~nm}$. However until the percolation network has developed to the point where Drude response is sufficiently strong, the spectral shapes differ little with separation for a given nanolayer, they just shift up or down across most of the solar spectrum range. Then thin film models using the optical constants and layer thicknesses evaluated before a mirror was added, describe the 3-layer optical response accurately. This means no evidence was found for any type of hybrid coupling between either of the two strong localised surface plasmon modes found in each nano-layer and the mirror, for the $5 \mathrm{~nm}$ and $6 \mathrm{~nm} \mathrm{Ag}$ masses at any spacing used. For $7 \mathrm{~nm}$ this is also so for spacings down to $20 \mathrm{~nm}$, but at lower spacings, a slight modulation of what the 3-layer models predict is observed in the data. The most surprising outcome, initially in this study, was that the nano-layer film with the largest Drude response gave the strongest hint that new effects were starting to happen. Since the range where differences arise is where the anomalous or hot-spot resonances lie, it is tempting to assume that they are responsible. However, their A values are actually weaker in the $7 \mathrm{~nm}$ base layer than in the $5 \mathrm{~nm}$ and $6 \mathrm{~nm}$ bases.

"A" values for the strength of any pre-mirror local plasmonic mode, as found in far field optics can be misleading as a basis for arguments on the likelihood of hybrid coupling. This is because the spectral densities given by parameter $A_{i}$ depend on two contributions, the fill factor $f_{i}$ of a the particular class of nanostructure defining each resonance peak and the average strength of the individual induced dipoles $p_{i}$ within that class. It is the strength $p_{i}$ of individual dipoles that will determine whether hybrid modes arise. One could thus have a situation where $f_{i}$ decreases and dipole strength $p_{i}$ increases, so that hybrid modes arise even though fill factor is decreasing. This will depend on the type of nanostructure involved in resonance $i$. It is unlikely for isolated nanoparticles, but may occur for the hot-spot resonances, which arise in the percolation transition zone. Their strength depends on local structural anomalies, or values of metal-to metal gaps, which decrease as more mass is added. This eventually leads to a fall in $f_{i}$ (hot spot) [3]. Both metal-in-metal or Babinet's core-shell resonant systems [3] and hot spots in nanowire networks [12] are examples. More insights into full 3-dimensional details of the base layer nanostructure, will be needed to help determine which hybrid modes are more likely to occur in a given nanostructure/spacer/mirror system. In the 3-layer system at percolation, Babinet's core shell effects may arise just in the initial nano-Ag plane, or between it and the mirror, if the mirror is not flat.

However, it is clear from this study that higher index spacer layers must be under $10 \mathrm{~nm}$ in thickness, to see strong evidence of hybrid modes and an associated breakdown of standard thin film models. The extent of evanescent fields around induced dipoles will play a key role in initiating such breakdown. It is quite sensitive to the $n$ value of the surrounding medium, being more tightly bound as $n$ increases. Thus, studies as a function of spacer refractive index, for spacer thicknesses in the range $6 \mathrm{~nm}$ to $15 \mathrm{~nm}$, should be of interest and value. A better understanding of evanescent field profiles, when metal nanoparticles or parts of the percolating network get very close as in all of the films in this study, would be of use in assessing which structures will be of most use. It is possible that in the narrow nano-gaps within the base films used here, near field spread tends to be more compressed within the film plane than for isolated nanoparticles, making any hybrid coupling across the spacer less likely. Much of this involves greater insights into the 
origins of the hot-spot or anomalous resonances, which are now known to be ubiquitous in growing metal films, from the onset of percolation until only a Drude term exists. What is fascinating is that for a given metal, they always cluster around a fixed energy. For silver this is close to $1 \mathrm{eV}$. What does vary for these anomalies is the broad width of this resonance, which we assume links to the spread of fine structural details between hot spots. Total resonant width narrows considerably as mass is added, in our case from $1.5 \mathrm{eV}$ at $5 \mathrm{~nm}$ to $1 \mathrm{eV}$ at $7 \mathrm{~nm}$, implying structural complexity decreases as one moves to a network with less voids and then to a dense film.

\section{CONCLUSION}

Nanostructure/spacer/mirror thin film systems, whether they manifest hybrid modes or not, promise enhanced ability to modify and control spectral absorptance over restricted wavelength ranges, as required for thermal output control via spectral emittance $\mathrm{e}(\lambda)$ or control of thermal input from irradiance via spectral absorptance $\mathrm{a}(\lambda)$, with $\mathrm{a}(\lambda)=\mathrm{e}(\lambda)$. Thus, potential applications are considerable. In general, thin film models using effective refractive indices for the nanocomposite layer suffice for design. To open up even wider variations and control options in spectral response, spacer layers will need in general to be thinner than $10 \mathrm{~nm}$ unless very low indices are used for the spacer material. Then hybrid modes or Fano resonances of various types will be possible. The enhanced absorptance found in this study is not due to a Fano effect, but simply phase modulation within the cavity between the mirror and nano-layer.

\section{Acknowledgement}

F. Bonnie is being supported by an Australian Postgraduate Scholarship.

\section{REFERENCES}

[1] Chopra, K.L.,[Thin film Phenomena], McGraw Hill, New York (1969).

[2] Smith, G.B., Maaroof, A.I. and Cortie, M.B., "Percolation in nanoporous gold and the Principle of Universality from two dimensions to hyperdimensions", Phys. Rev B., 78, 165418 (11pp) (2008) .

[3] Earp, A.A. and Smith, G.B., "Evolution of plasmonic optical response in growing silver thin films with prepercolation non-local conduction and emittance," J. Phys. D: Appl. Phys. 44, 255102 (8pp) (2011).

[4] Bermel, P., Ghebrebrhan, M., Harradon, M., Yeng, Y.X., Celanovic, I., Joannopoulos, J.D. and Soljacic, M., "Tailoring photonic metamaterial resonances for thermal radiation" Nano Express, Nanoscale Research Letters, $6: 549$ $1-5$ (2011).

[5] Smith, G.B. and Granqvist, C.G., [Green Nanotechnology: Solutions for Sustainability and Energy in the Built Environment], CRC Press, Boca Raton (2010).

[6] Rephaeli, E., Raman. A., and Fan, S., "Ultrabroadband Photonic Structures To Achieve High-Performance Daytime Radiative Cooling," Nano Lett. 13, 1457-1461 (2013).

[7] Gentle, A.R. and Smith, G.B., "Radiative heat pumping from the earth using surface phonon resonant nanoparticles," NanoLetters, 10, 373-37 (2010).

[8] Gentle, A.R., Aguilar, J.L.C. and Smith, G.B., "Optimized cool roofs: integrating albedo and thermal emittancewith R-Value," Solar Energy Materials and Solar Cells 95 3207-3215 (2011).

[9] Molesky, S., Dewalt, C.J. and Jacob, Z., "High temperature epsilon-near-zero and epsilon-near-pole metamaterial emitters for thermophotovoltaics," Optics Express A96-110 (2013).

[10] Ciracì, C., Hill, R.T., Mock, J. J., Urzhumov, Y., Fernández-Domínguez, A. I.,

Maier,3, S. A., Pendry, J. B., Chilkoti, A. and Smith, D. R., "Probing the Ultimate Limits of Plasmonic Enhancement," Science 337, 1072 (2012); DOI: 10.1126/science.1224823

[11] Maaroof, A. I. and Sutherland, D. S., "Optimum plasmon hybridization at percolation threshold of silver films near metallic surfaces, J. Phys. D: Appl. Phys. 43405301 (5pp) (2010).

[12] Pavaskar, P., Hsu, I-K., Theiss, J., Hung, W.H. and Cronin, S.B., "A microscopic study of strongly plasmonic Au and Ag island thin films," J. App. Phys. 113, 034302 (2013); doi: 10.1063/1.4775784.

[13] Smith, G.B. and Earp, A.A., "Metal-in-metal localised surface plasmon resonance," Nanotechnology 21015203 (2010).

[14] Liu, X., Tyler,T., Starr, A.F., Jokerst, N.M. and Padilla, W.J., "Taming the Blackbody with Infrared Metamaterials as Selective Thermal Emitters,” Phys. Rev. Lett. 107, 045901 (2011).

[15] Leitner, A., Zhao, Z., Brunner, H., Franz R. Aussenegg, F.R. and Wokaun, A., "Optical properties of a metal island film close to a smooth metal surface," Applied Optics 32, 102-110 (1993). 